\title{
Plasmocytome solitaire de la mandibule. À propos de deux cas
}

\section{RÉSUMÉ}

Zied BELLALAH

Résident en chirurgie maxillo-faciale, Service de chirurgie maxillo-faciale, CHU Sahloul,

Route Ceinture, Cité Sahloul,

Sousse, Tunisie.

\section{Abdelfateh SLAMA}

Assistant hospitalo-universitaire en chirurgie maxillo-faciale, Service de chirurgie maxillo-faciale, CHU Sahloul,

Sousse, Tunisie.

\section{Samia AYACHI}

Assistant hospitalo-universitaire en chirurgie maxillo-faciale, Service de chirurgie maxillo-faciale, CHU Sahloul,

Sousse, Tunisie.

\section{Soumaya RAMMEH}

Maître de conférence agrégé en anatomie-pathologie, Service d'anatomie-pathologie, CHU F. Hached,

Sousse, Tunisie.

Kaouther MRAD-DALY

Maître de conférence agrégé en radiologie,

Service de radiologie,

CHU Sahloul,

Sousse, Tunisie.

\section{Habib Khochtali}

Professeur, chef de service, Service de chirurgie maxillo-faciale, CHU Sahloul,

Sousse, Tunisie.
Le plasmocytome solitaire est une tumeur maligne caractérisée par une prolifération monoclonale de plasmocytes. II atteint préférentiellement le rachis et le pelvis. Sa localisation mandibulaire est exceptionnelle. Les auteurs rapportent deux observations et rappellent les aspects radiocliniques, thérapeutiques et évolutifs de cette pathologie rare.

Il s'agissait d'un homme et d'une femme âgés respectivement de 38 et 70 ans qui ont consulté pour des tumeurs mandibulaires étiquetées histologiquement plasmocytome. Le bilan d'extension tumorale était négatif, dans les deux cas, confirmant son caractère solitaire. Une exérèse tumorale a été réalisée chez les deux patients.

L'évolution était marquée par une transformation myélomateuse dans le premier cas nécessitant une polychimiothérapie adjuvante. plasmocytome

- solitaire

- mandibule 


\section{Introduction}

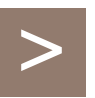

Le plasmocytome solitaire osseux est une tumeur maligne caractérisée par une prolifération monoclonale de lymphocytes B plus ou moins différenciés [1]. L'absence de sécrétion d'immunoglobulines (Ig) monoclonales et de dissémination de la prolifération définissent le caractère solitaire

\section{Observation 1}

Il s'agit du patient R.S. âgé de 38 ans qui a consulté pour une tuméfaction préauriculaire gauche évoluant depuis trois mois. L'examen exobuccal a révélé une masse ferme de $2,5 \mathrm{~cm}$, indolore et semblant faire corps avec le plan osseux sous-jacent, sans paralysie faciale ni trouble de la sensibilité. En endobuccal, on a noté un trouble de l'articulé dentaire sans limitation de l'ouverture buccale. La radiographie panoramique a montré une image lytique monogéodique allant de l'échancrure sigmoïde au condyle mandibulaire gauche (fig. 1). du plasmocytome. La localisation mandibulaire du plasmocytome solitaire est exceptionnelle.

À partir de deux observations et d'une revue de la littérature, les auteurs rappellent les aspects cliniques, radiologiques, thérapeutiques et évolutifs de cette pathologie rare.

L'examen tomodensitométrique a objectivé une ostéolyse de la branche montante et du condyle mandibulaire gauche avec rupture des deux corticales et sans extension aux parties molles (fig. 2). Le patient a eu une exérèse tumorale emportant le condyle gauche. L'examen anatomopathologique après étude immunohistochimique a conclu à un plasmocytome de phénotype IgM kappa, complètement réséqué. Un bilan d'extension a été pratiqué (radiographie du squelette, myélogramme, bilan biologique) et n'a montré aucune ano-
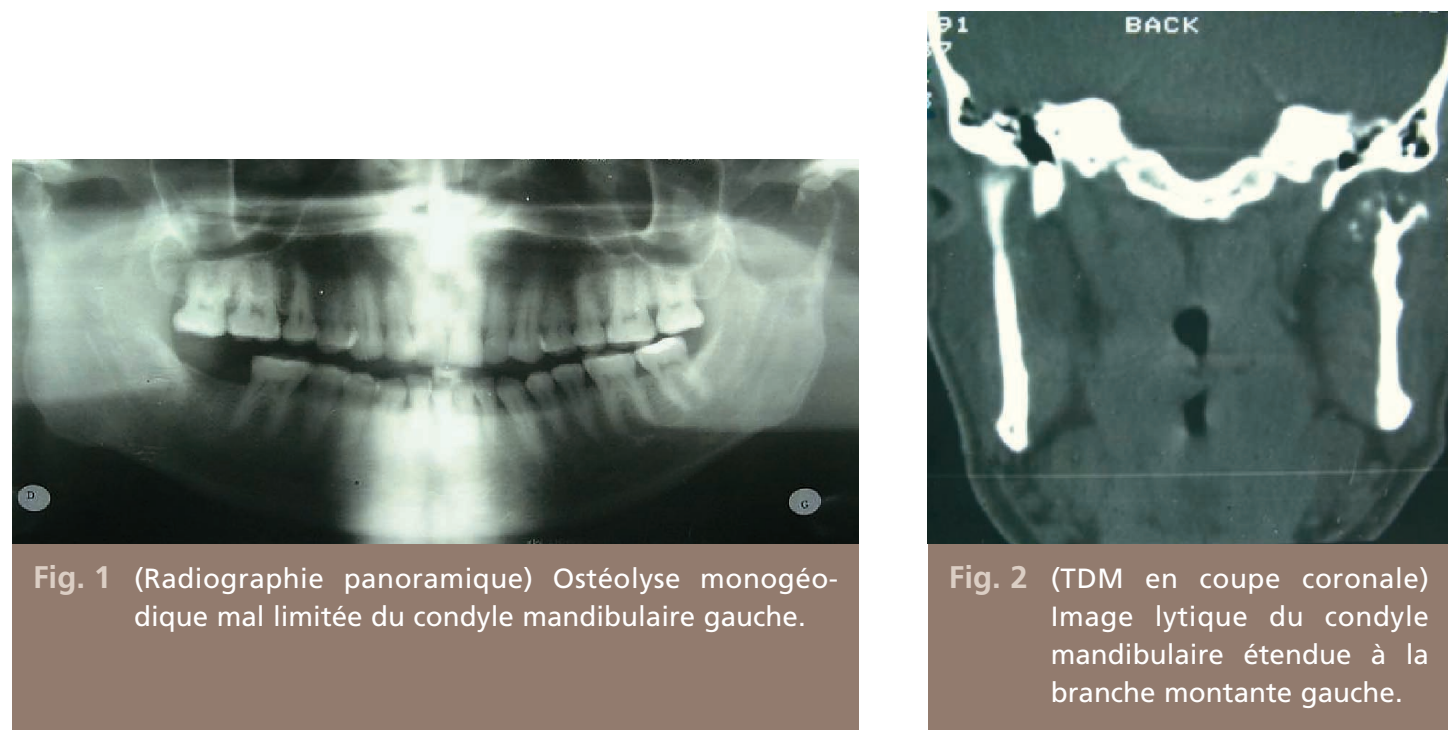


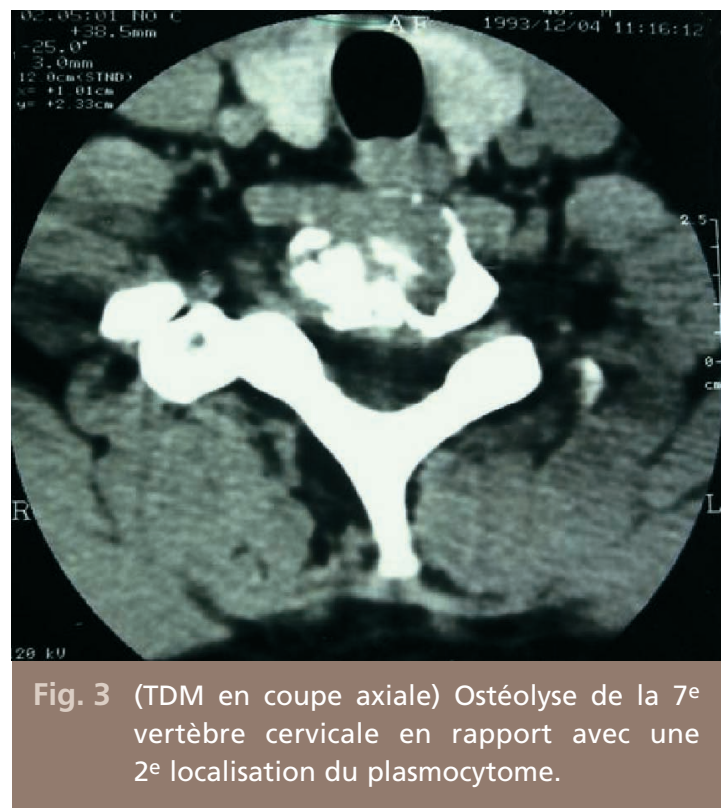

malie confirmant le caractère solitaire du plasmocytome mandibulaire.

Après un recul de 20 mois, le patient avait présenté des névralgies cervicobrachiales droites en rapport avec une deuxième localisation du plasmocytome au niveau de la $7 \mathrm{e}$ vertèbre cer-

\section{Observation 2}

Madame C.L. âgée de 70 ans avait consulté pour une tuméfaction mandibulaire gauche évoluant depuis quatre mois. L'examen exobuccal avait objectivé une tuméfaction osseuse de l'angle mandibulaire gauche, sensible, de $3 \mathrm{~cm}$ de grand axe et associée à une hypoesthésie labio-mentonnière homolatérale. À l'examen endobuccal, on a noté une édentation totale et un comblement vestibulaire en regard de la région molaire inférieure gauche associé à une soufflure de la corticale interne. La radiographie panoramique a montré une ostéolyse multigéodique à contours nets étendue de la branche horizontale à la branche vicale (fig. 3). La scintigraphie osseuse avait montré plusieurs foyers hyperfixants traduisant des localisations multiples. Le patient a eu une polychimiothérapie, puis il a été perdu de vue.

montante gauche respectant le coroné et le condyle. La tomodensitométrie a objectivé une image multiloculaire étendue avec soufflure et rupture des corticales par endroits ainsi qu'une extension aux parties molles adjacentes (fig. 4). L'imagerie par résonance magnétique a révélé une extension tumorale au-delà des corticales avec infiltration du muscle ptérygoïdien médial (fig. 5). Le diagnostic d'améloblastome ou de sarcome améloblastique a été évoqué. Il a été réalisé une résection mandibulaire interruptrice allant de la région parasymphysaire à la branche montante gauche. L'examen anatomopathologique complété par une 

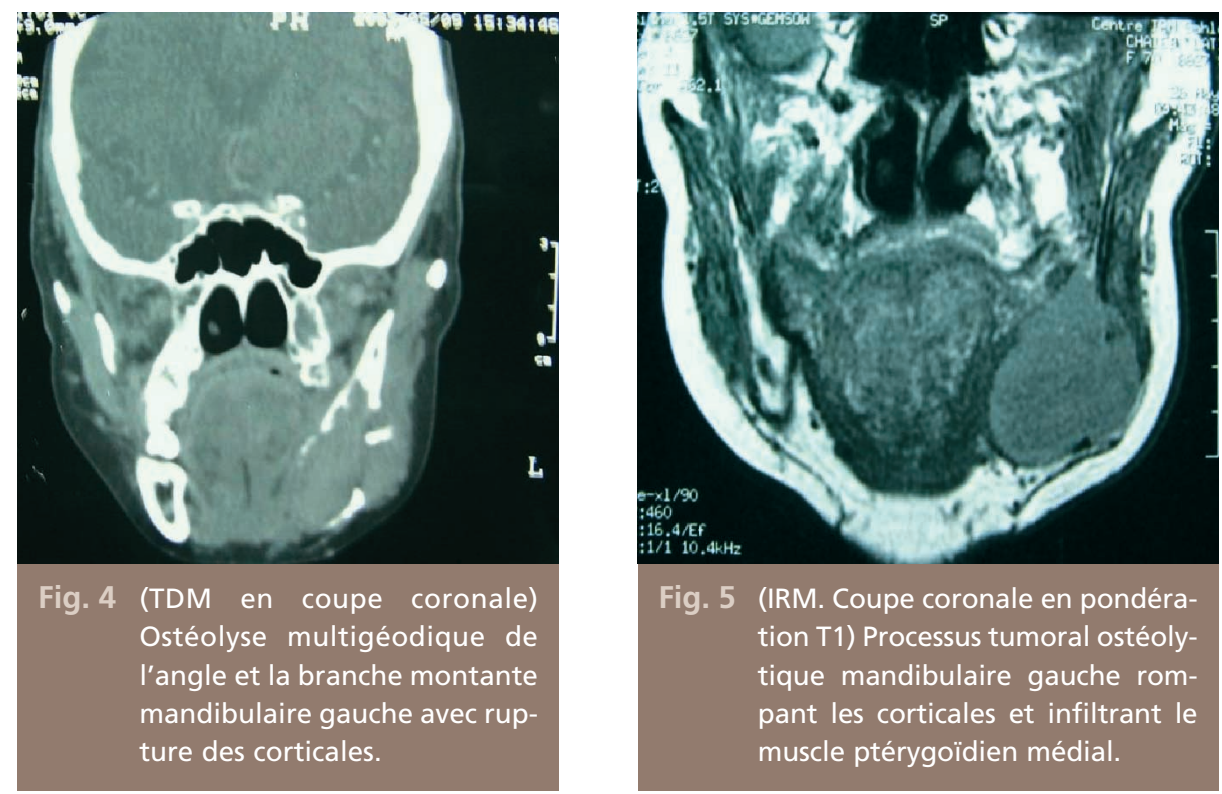

Fig. 5 (IRM. Coupe coronale en pondération T1) Processus tumoral ostéolytique mandibulaire gauche rompant les corticales et infiltrant le muscle ptérygoïdien médial.

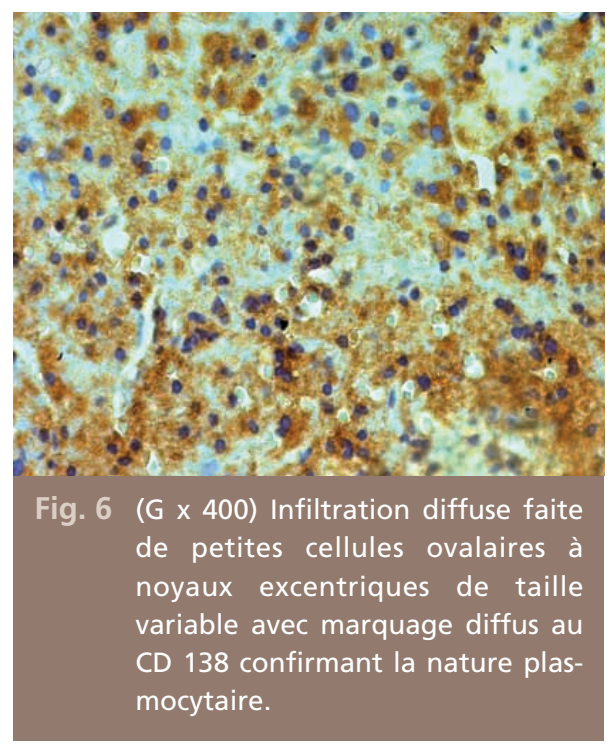

étude immunohistochimique avait confirmé le diagnostic de plasmocytome (fig. 6). Le bilan d'extension tumoral était négatif et a conclu à

\section{Discussion}

Parmi les tumeurs plasmocytaires de l'extrémité céphalique, on distingue le myélome multiple, le plasmocytome extra-médullaire et le un plasmocytome solitaire. Les suites opératoires étaient simples et la patiente a eu une polychimiothérapie adjuvante.

plasmocytome osseux solitaire. Le myélome multiple ou maladie de Kahler représente $93 \%$ des myélomes plasmocytaires [2]. Le 
plasmocytome osseux solitaire est plus rare et n'en représente que $4 \%$.

II touche préférentiellement le rachis et les os longs [3]. Les localisations crâniennes et maxillaires représentent $4 \%$ des cas [4]. La mandibule est plus fréquemment atteinte que le maxillaire [5], particulièrement au niveau de la région prémolo-molaire qui est riche en cellules hématopoïétiques [6]. L'atteinte masculine est prédominante [7] et l'âge moyen de survenue est de 50 ans [5].

La présentation clinique du plasmocytome solitaire de la mandibule (PSM) n'est pas spécifique.

La tuméfaction faciale, la mobilité dentaire et les troubles sensitifs sont les signes cliniques les plus fréquents. Rarement, le PSM peut être découvert à l'occasion d'une fracture pathologique, d'un saignement postextractionnel ou d'un trouble de l'articulé dentaire pour les localisations condyliennes [6]. L'aspect radiologique est celui d'une image ostéolytique pouvant être mono- ou multiloculaire [8]. Les lésions radio-opaques sont exceptionnelles [9]. La tomodensitométrie permet d'affiner l'image radiologique et d'étudier l'extension vers les corticales et les parties molles. Ces aspects radiocliniques du plasmocytome solitaire de la mandibule sont variables et posent le problème de diagnostic différentiel avec les tumeurs kystiques et pseudokystiques ainsi que les métastases des tumeurs malignes [8].

Le diagnostic de certitude du PSM repose sur l'examen anatomopathologique complété par I'immunohistochimie [10].

Un bilan d'extension est nécessaire pour affirmer le caractère solitaire du plasmocytome.

Ce bilan comporte une scintigraphie osseuse et/ou des radiographies du squelette, un myé- logramme et un bilan biologique (électrophorèse et immunoélectrophorèse des protéines sériques, bilan calcique, hémogramme et recherche de protéinurie de Bences Jones) [8]. Le diagnostic de plasmocytome solitaire sera porté devant la présence d'une prolifération plasmocytaire localisée à la mandibule avec normalité de la cytologie médullaire et du bilan biologique (absence de sécrétion monoclonale) [11].

Le principal risque évolutif du plasmocytome solitaire est la survenue d'un myélome multiple ou maladie de Kahler avec des délais variables allant de quelques mois à dix ans [12]. La fréquence de cette transformation myélomateuse varie entre 45 et $75 \%$ [13]. Pour Maalej et al. [14], la survenue d'une dissémination tumorale dans un délai inférieur à deux ans devrait mettre en doute le diagnostic de plasmocytome solitaire.

Le traitement du plasmocytome solitaire de la mandibule repose sur la chirurgie et/ou la radiothérapie [14, 15]. L'exérèse tumorale dépend de son extension locale. La radiothérapie vise à stériliser les foyers résiduels après une chirurgie afin de limiter les récidives. Elle doit être d'au moins 50 Gys [5]. Des doses insuffisantes constituent un facteur de récidive locale [11]. La place de la chimiothérapie dans le traitement du plasmocytome solitaire mandibulaire est discutée et elle est indiquée en cas de survenue de myélome multiple $[8,16,17]$.

L'évolution des PSM reste dominée par la survenue de myélome multiple dont le pronostic est sévère.

Ainsi une surveillance régulière (clinique, radiologique et biologique) s'impose et permet de guetter les récidives et/ou la transformation myélomateuse. 


\section{Bibliographie}

1. Muzio L, Pannone G,

Bucci P.

Early clinical diagnosis

of solitary plasmocytoma

of the jaws:

a case report

with a six year follow-up.

Int J Oral Maxillofac Surg

2001:30:558-560.

2. Gonzales J, Elizondo J, Trull JM, De Torres I.

Plasma-cell tumors

of the condyle.

Br J Oral Maxillofac Surg

1991;29:274-276.

3. Millesi $\mathrm{W}$, et al.

Solitary plasmocytoma

of the mandible:

A combined approach

for treatment

and reconstruction.

Int J Oral Maxillofac Surg 1997;26:295-298.

4. Bataille R, Sany J, Serre H. Plasmocytomes apparemment solitaires des os:

aspects cliniques

et pronostiques.

Nouv Presse Med

1981;10:407-411.

5. Ilankovan V, Moos K.F,

El Attar A.

Intramedullary

plasma cell tumors.

Int J Oral Maxillofac Surg

1990;19:323-326.

6. Florencio $\mathrm{M}$, Jose $\mathrm{L}$,

Gil-Diez, Campano F.J,

Jose R, Del Hoyo A.

Mandibular lesion

as the first evidence

of Multiple Myeloma.

J Craniomaxillofac Surg

1989;17:315-317.
7. Shoemaker E.I,

Romano A.J,

Gado M, Hodges F.J.

Neuroradiology case

of the day:

Plasmocytoma

of the skull vault.

Am J Roentgenol

1989;152:1333-1338.

8. Collangettes-Peyrat $D$, Baudet-Pommel M, Fonck $Y$, Meyniel P. Plasmocytome solitaire de la mandibule : étude d'un cas. Actual Odonto Stomatol 1990;169:175-183.

9. Mustoe T.H, Fried M.P, Goodman M.L, Kelly J.H, Strome M.

Osteosclerotic plasmocytoma of maxillary bone (orbital floor).

J Laryngol Otol 1984;98:929-938.

10. Khochtali $\mathrm{H}$, Yacoubi M.T, Bouzaiene M, Abassi-Bakir D, Ben Hammouda M, Krifa $\mathrm{H}$.

Plasmocytome solitaire :

À propos d'un cas de localisation cranio-faciale. Rev Stomatol Chir Maxillofac 1992;93:377-380.

11. Khochbati $L$, et al. Solitary Plasmocytoma. A propos of a case of craniofacial involvement.

Cancer Radiother 2004;8:70-74.

12. Liebross $\mathrm{RH}, \mathrm{Ha} \mathrm{CS}$, Cox JD, Weber D, Delasalle $\mathrm{K}$, Alexanian R.

Solitary bone plasmocytoma:

Outcome and prognostic factors following radiotherapy. Int J Radiat Oncol

Biol Phys

1998:41:1063-1067.

13. Meyer J.E, Schulz M.D. Solitary myeloma of bone:

Review of 12 cases.

Cancer 1974;34:438-440.

14. Maalej M, Moalla M.

Plasmocytome

solitaire osseux :

Problèmes nosologiques et thérapeutiques.

Tunis Med 1989;67:661-664.

15. Woodruff RK, Whittle JM, Malpas JS.

Solitary plasmocytoma.

Cancer 1979;43:2340-2343.

16. Bataille R.

Localized plasmocytomas.

Clin Haematol 1982;11:11-122.

17. Grellet M. Tumeur plasmocytaire maligne isolée.

Rev Stomatol

Chir Maxillofac 1982;83:45-46. 


\section{SUMMARY}

\section{Solitary plasmocytoma of the mandible: 2 cases reports}

\author{
Zied BELLALAH， \\ Abdelfateh SLAMA, \\ Samia AYACHI, \\ Soumaya RAMMEH, \\ Kaouther MRAD-DALY, \\ Habib KHOCHTALI
}

Keywords
- plasmocytoma
- solitary
- mandible

Solitary plasmocytoma is a malignant tumour of differentiated B-cells. The most common sites are vertebral bodies and the pelvis. Its location in the mandible is exceptional.

This article describes the case of a 38-year-old man and a 70-year-old woman presenting a plasmocytoma of the mandible. The presence of systematic involvement was excluded and a surgical resection was performed in the 2 cases.

In the first case, plasmocytoma developped into a multiple myeloma treated by chemotherapy. We propose to analyse the clinical presentation, the radiographic appearance and the management of these rare entities.Anemias often show oral symptoms that must be known by the dental practitioner, because they may allow an early detection.

We report two cases of patients consulting for oral lesions suggestive of anemia. Blood examinations confirm the first suspicion.

A review of the subject is presented, with its clinical implications.

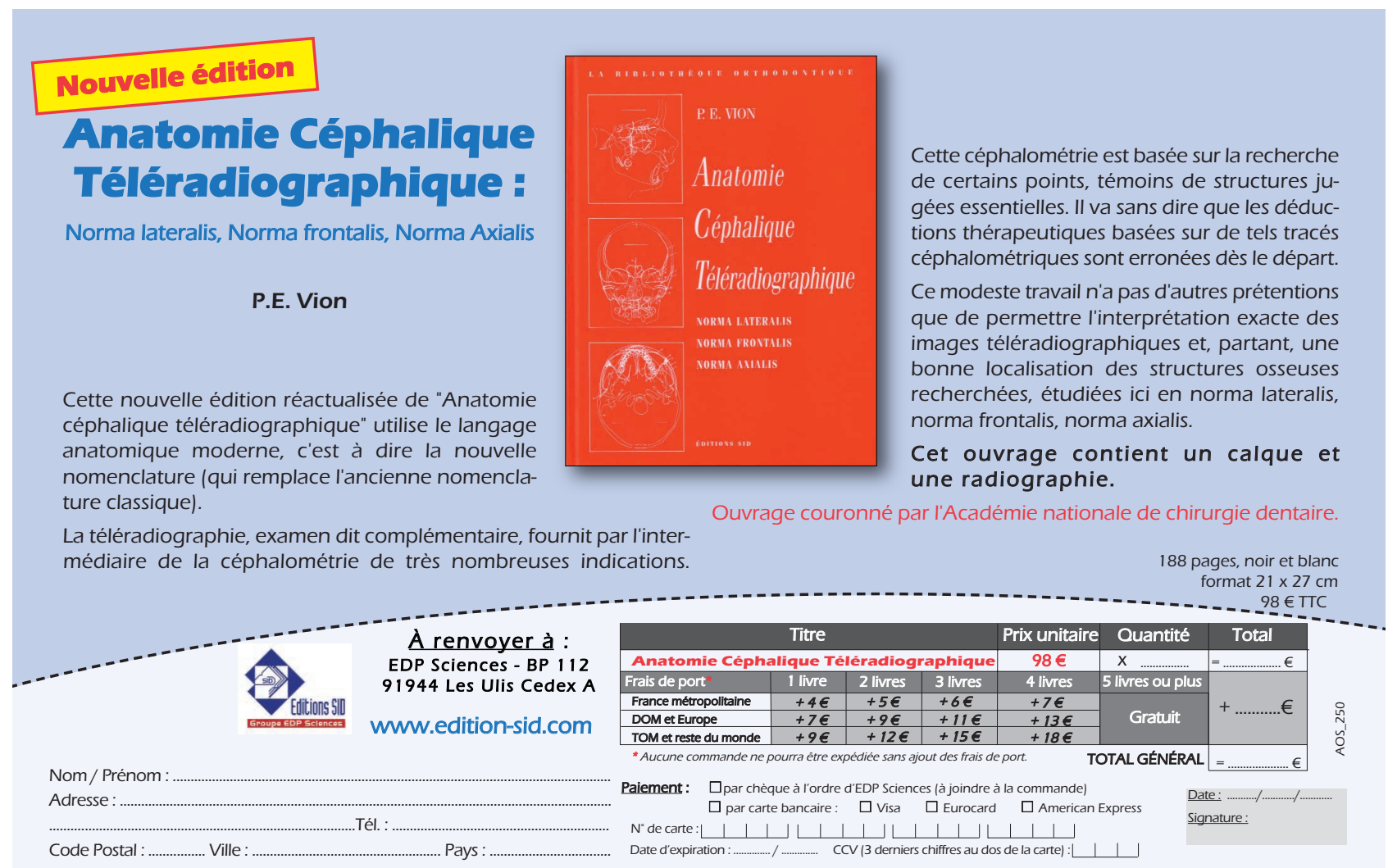

